\title{
Guest Editorial: Special section on "Foundations of resilience for networked robotic systems"
}

\author{
Amanda Prorok $^{1} \cdot$ Brian M. Sadler ${ }^{2} \cdot$ Magnus Egerstedt $^{3} \cdot$ Vijay Kumar $^{4}$
}

Published online: 28 November 2018

(c) Springer Science+Business Media, LLC, part of Springer Nature 2018

This special section of Autonomous Robots is devoted to the topic of resilience. The timeliness of this topic is granted by our aspirations to solve increasingly hard problems in complex, contested, and adversarial environments. We wish to see teams of robots operating in such unstructured environments through resilient behaviors that allow them to embrace changes, to endure unexpected disruptions and recover swiftly.

Yet, classical approaches to fault tolerance focus on rejecting changes and disturbances, and require individual robots to be over-provisioned with redundant capabilities and resources. This special section will explore an alternative research question: How are we to create perception, inference, planning, and communication modules for individual robots, such that resilience is achieved at the team level? The three articles in this section explore new algorithmic and mathematical foundations towards answering this question. They cover three key problem domains: resilient distributed estimation, robust connectivity maintenance, and distributed planning in heterogeneous networks.

The first article, by Mitra et al., "Resilient distributed state estimation with mobile agents: overcoming Byzantine adversaries, communication losses, and intermittent measurements", develops a resilient, fully-distributed, and provably correct state estimation algorithm under adversarial settings. The authors develop a simple switched linear observer for dealing with the issue of time-varying measurements, as well as resilient filtering techniques for dealing with a certain number of malicious or Byzantine agents, subject to time-varying communication patterns among the agents.

\footnotetext{
Amanda Prorok

asp45@cam.ac.uk

University of Cambridge, Cambridge, UK

2 Army Research Laboratory, Adelphi, USA

3 Georgia Institute of Technology, Atlanta, USA

4 University of Pennsylvania, Philadelphia, USA
}

In our second paper, "Robust Connectivity Maintenance for Fallible Robots", the authors Panerati et al., present a controller for connectivity maintenance in the presence of faults. Based on graph theoretic foundations, they estimate a node 'vulnerability' level, and derive a controller that increases the number of links to vulnerable nodes. Experimental trials test two types of failure (communications-related and hardwarerelated), and show that it is possible to preserve and even improve robot-to-robot connectivity in the presence of disruptions.

In our third paper, "Real-time distributed non-myopic task selection for heterogeneous robotic teams", the authors Smith et al., present a real-time, probabilistic and distributed task assignment algorithm for heterogeneous robot teams. The work leverages a novel representation of the multi-robot action space, enabling individual robots to coordinate actions in real-time and collect rewards in discrete environments. A series of fielded hardware trials show that their coordination method significantly increases the cumulative team reward. This method can be applied across a wide range of tasks, robot team compositions, and reward functions.

Collectively, these three articles provide key ideas within the topic of resilience. Although our selection represents but a thin slice, we hope it will spark further efforts that address this urgent and important area of autonomous robots.

Signatures:

Amanda Prorok

Brian M. Sadler

Magnus Egerstedt

Vijay Kumar

Publisher's Note Springer Nature remains neutral with regard to jurisdictional claims in published maps and institutional affiliations. 